\title{
Typing of Ochrobactrum anthropi clinical isolates using automated repetitive extragenic palindromic-polymerase chain reaction DNA fingerprinting and matrix-assisted laser desorption/ ionization-time-of-flight mass spectrometry
}

\author{
Angela Quirino ${ }^{1}$, Giovanna Pulcrano², Linda Rametti ${ }^{1}$, Rossana Puccio ${ }^{1}$, Nadia Marascio ${ }^{1}$, Maria Rosaria Catania², \\ Giovanni Matera', Maria Carla Liberto ${ }^{1 *}$ and Alfredo Focà ${ }^{1}$
}

\begin{abstract}
Background: Ochrobactrum anthropi (O. anthropi), is a non-fermenting gram-negative bacillus usually found in the environment. Nevertheless, during the past decade it has been identified as pathogenic to immunocompromised patients. In this study, we assessed the usefulness of the automated repetitive extragenic palindromic-polymerase chain reaction (rep-PCR-based DiversiLab ${ }^{\mathrm{TM}}$ system, bioMèrieux, France) and of matrix-assisted laser desorption/ ionization-time-of-flight (MALDI-TOF MS) for typing of twentythree $O$. anthropi clinical isolates that we found over a four-months period (from April 2011 to August 2011) in bacteriemic patients admitted in the same operative unit of our hospital. Pulsed-field gel electrophoresis (PFGE), commonly accepted as the gold standard technique for typing, was also used. Analysis was carried out using the Pearson correlation coefficient to determine the distance matrice and the unweighted pair group method with arithmetic mean (UPGMA) to generate dendogram.
\end{abstract}

Results: Rep-PCR analysis identified four different patterns: three that clustered together with $97 \%$ or more pattern similarity, and one whose members showed $<95 \%$ pattern similarity. Interestingly, strains isolated later (from 11/06/ 2011 to 24/08/2011) displayed a pattern with 99\% similarity. MALDI-TOF MS evaluation clustered the twentythree strains of $O$. anthropi into a single group containing four distinct subgroups, each comprising the majority of strains clustering below 5 distance levels, indicating a high similarity between the isolates.

Conclusions: Our results indicate that these isolates are clonally-related and the methods used afforded a valuable contribution to the epidemiology, prevention and control of the infections caused by this pathogen.

Keywords: Ochrobactrum anthropi, rep-PCR fingerprinting, MALDI-TOF MS, Strain typing

\section{Background}

Ochrobactrum anthropi (O. anthropi) is a non-fermenting, aerobic, gram-negative bacillus that exhibits widespread resistance to $\beta$-lactam antibiotics [1,2] and is able to colonize a variety of environments, namely soil, plants, insects, animals and humans [3]. Reports of opportunistic/ nosocomial infections caused by $O$. anthropi have been

\footnotetext{
*Correspondence: mliberto@unicz.it

'Institute of Microbiology, Department of Health Sciences, "Magna Graecia" University, Viale Europa, Catanzaro, Italy

Full list of author information is available at the end of the article
}

increasing over the last decade [4-6], and the ability of $O$. anthropi to adhere to silicone may play a role in catheter-associated infections [6,7]. Furthermore, O. anthropi populations may adapt in response to habitat and host interactions, as previously described in human clinical isolates $[3,8]$. In the human infection: a catheter-associated bacteremia caused by $O$. anthropi has been shown [1]. In literature, the infections due to $O$. anthropi involved catheter related bacteremia, whereas endophalmitis, urinary infections, meningitis, endocarditis, hepatic, pelvic and

\section{()


pancreatic abscess often as monomicrobial infection have been reported $[1,4,6,9]$

According to their habitat, the population structure of $O$. anthropi varied. For example, biological and genomic microdiversity was higher in bulk soil than in the rhizoshere [10,3]. Authors related this difference in diversity level to the expansion of clones adapted to metabolites produced by rhizodeposition [3].

Among the few publications regarding the known methods for typing of $O$. anthropi relevant papers are those from Romano et al., 2010 [3] dealing with MLST and PFGE. Also, Bathe et al., 2006 [11] described the rep-PCR of $O$. anthropi (however with a instrument different than Diversilab, bioMerieux). Finally, Bizzini et al., 2010 [12] reported on Maldi-TOF characterization of $O$. anthropi.

The different typing methods used, mainly rep-PCR and Maldi-TOF, in terms of time, accuracy and costs may allow to obtain more timely, accurate results with higher resolution among the different strains involved in hospital outbreak. When this infection did occur in our hospital, we set out to study the identification and typing of the twentythree $O$. anthropi strains. Strain typing was carried out by automated repetitive extragenic palindromicpolymerase chain reaction (rep-PCR-based DiversiLab ${ }^{\mathrm{TM}}$ system, bioMèrieux, France) and by pulsed-field gel electrophoresis (PFGE). Proteome profiling was performed through matrix-assisted laser desorption/ionizationtime-of-flight (MALDI-TOF MS).

The application of accurate and more powerful techniques, used for typing, should be encouraged for monitoring the spread of bacteria and nosocomial infection control.

\section{Methods}

Bacterial strains and microbiological methods

During a 4-month period (from April 2011 to August 2011) 23 O. anthropi strains were isolated from samples of 19 patients admitted to the Catanzaro University Hospital (Italy) Oncology O.U. Samples were taken as part of standard patient care and all procedures were approved by the local ethics committee at the Medical Faculty of the University "Magna Graecia" of Catanzaro, which are in compliance with Declaration of Helsinki (59th WMA General Assembly, Seoul, October 2008).

During stay in hospital, all patients, which presented severe background disease, mainly neoplasia, showed mild clinical signs of sepsis. We therefore performed blood cultures by BacT/Alert 3D system (bioMèrieux, Clinical Diagnostics, France), detecting 18 isolates from 18 positive blood cultures drawn from the central venous catheter (CVC) and 5 isolates from positive catheter tip cultures (Table 1). The strains were conventionally identified by typical Gram stain morphology and biochemical testing (Vitek-2, bioMèrieux, France). Antibiotic sensitivity
Table 10 . anthropi strains isolated from patients admitted to the Oncology O.U.

\begin{tabular}{|c|c|c|c|}
\hline Strain ID & Patient ID & Isolation location & Date of isolation \\
\hline CZ1403 & 1 & Blood & $26 / 04 / 2011$ \\
\hline CZ1424* & 2 & Blood & $17 / 05 / 2011$ \\
\hline CZ1427* & 3 & Blood & $19 / 05 / 2011$ \\
\hline CZ1425 & 4 & Blood & $20 / 05 / 2011$ \\
\hline CZ1429* & 3 & Catheter tip & $25 / 05 / 2011$ \\
\hline CZ1433 & 5 & Blood & $06 / 06 / 2011$ \\
\hline CZ1439 & 6 & Blood & $06 / 06 / 2011$ \\
\hline CZ1442 & 7 & Blood & $09 / 06 / 2011$ \\
\hline CZ1443* & 2 & Catheter tip & $09 / 06 / 2011$ \\
\hline CZ1449* & 3 & Catheter tip & $11 / 06 / 2011$ \\
\hline CZ1454 & 8 & Blood & $17 / 06 / 2011$ \\
\hline CZ1458 & 9 & Blood & $20 / 06 / 2011$ \\
\hline CZ1460 & 10 & Blood & $21 / 06 / 2011$ \\
\hline CZ1474 & 11 & Blood & $29 / 06 / 2011$ \\
\hline CZ1476 & 12 & Blood & $29 / 06 / 2011$ \\
\hline CZ1505 & 13 & Catheter tip & 07/07/2011 \\
\hline CZ1504* & 14 & Blood & $08 / 07 / 2011$ \\
\hline CZ1523* & 14 & Catheter tip & $14 / 07 / 2011$ \\
\hline CZ1532 & 15 & Blood & $15 / 07 / 2011$ \\
\hline CZ1519 & 16 & Blood & $19 / 07 / 2011$ \\
\hline CZ1541 & 17 & Blood & $20 / 07 / 2011$ \\
\hline CZ1552 & 18 & Blood & $26 / 07 / 2011$ \\
\hline CZ1573 & 19 & Blood & $24 / 08 / 2011$ \\
\hline
\end{tabular}

*strains isolated from the same patient.

was evaluated by Vitek System (bioMèrieux, France). To exclude Brucella misdiagnosis, the O. anthropi colonies of all isolates were tested with Brucella agglutinating sera (Brucella spp., Brucella abortus and Brucella melitensis).

\section{Rep-PCR-based DNA fingerprinting by the DiversiLab System}

For rep-PCR analysis, bacteria (23 clinical strains of $O$. anthropi, in addition to O. anthropi ATCC49188T and O. intermedium LMG3301T, kind gifts from Dr. Fabien Aujoulat, Universitè Montpellier, France) were grown on Columbia blood agar; DNA was extracted from a $10-\mu \mathrm{l}$ loopful of each $O$. anthropi colony, using an UltraClean Microbial DNA isolation kit (Mo Bio Laboratories, Carlsbad, CA). The extracted DNA was amplified using a DiversiLab Generic DNA fingerprinting kit (bioMèrieux, France), following the manufacturer's instructions. DiversiLab Rep-PCR was performed according to Treviño M. et al., 2011 [13]. Briefly, 50 ng of genomic DNA, $2.5 \mathrm{U}$ of AmpliTaq DNA polymerase, and $1.5 \mu \mathrm{l}$ of $10 \times$ PCR buffer (Applied Biosystems, Foster City, CA) were added to the appropriate rep-PCR master mix to achieve 
a total of $25 \mu \mathrm{l}$. Thermal cycling parameters were as follows: initial denaturation at $94^{\circ} \mathrm{C}$ for $2 \mathrm{~min}, 35$ cycles of denaturation at $94^{\circ} \mathrm{C}$ for $30 \mathrm{~s}$, annealing at $60^{\circ} \mathrm{C}$ for $30 \mathrm{~s}$, extension at $70^{\circ} \mathrm{C}$ for $90 \mathrm{~s}$, and final extension at $70^{\circ} \mathrm{C}$ for $3 \mathrm{~min}$. DNA concentration was measured by NANODROP 1000 Spectrophotometer (Thermo Scientific). The amplified product was stored at $-20^{\circ} \mathrm{C}$ until detection. Analysis of rep-PCR products was performed using a DiversiLab system, in which the amplified fragments of various sizes and intensities are separated and detected using a microfluidic LabChip by an Agilent 2100 Bioanalyzer (Agilent Technologies, Palo Alto, CA). The relatedness of the isolates was analysed by means of the DiversiLab software, version 3.4, using the Pearson correlation coefficient, to determine distance matrices, and the unweighted-pair group method with arithmetic mean (UPGMA). Further analysis was performed by the Kullback-Leibler distance correlation coefficient.

In general, "different" was defined as $<95 \%$ pattern similarity and 2 or more band differences for organisms defined as homogeneous, and three or more band differences for organisms defined as heterogeneous. "Similar" was defined as $>95 \%$ and $<97 . \%$ pattern similarity and one band difference for homogeneous organisms, or up to two band differences for heterogeneous organisms. "Indistinguishable" was defined as $>97 \%$ pattern similarity and no band differences, including no variation in the intensities of individual bands, although overall intensities may differ [13].

\section{Genotyping by PFGE}

The $23 O$. anthropi isolates were grown overnight and suspended in SE buffer ( $75 \mathrm{mM} \mathrm{NaCl}, 25 \mathrm{mM}$ EDTA, $\mathrm{pH}$ 7.5). The cell suspensions (4 McFarland units) were mixed with an equal volume of $2 \%$ low-melting point agarose, moulded into plugs at $4^{\circ} \mathrm{C}$, and lysed with lysis buffer (1\% N-lauryl sarcosine, $0.5 \mathrm{M}$ EDTA, pH 8) supplemented with Proteinase K $(500 \mu \mathrm{g} / \mathrm{mL})$. The DNA contained in each plug was digested by $20 \mathrm{U}$ of SpeI restriction enzyme in accordance with the manufacturer's instructions. Macrorestriction fragments were separated using the CHEF-DR III PFGE system (Bio-Rad) at $10^{\circ} \mathrm{C}$ for $20 \mathrm{~h}$, at a field strength of $6 \mathrm{~V} / \mathrm{cm}$, with an initial switch time of $5 \mathrm{~s}$ and a final switch time of $35 \mathrm{~s}$. A lambda ladder of phage DNA concatemers was used as a size marker. O. anthropi ATCC $49188 \mathrm{~T}$ and O. intermedium LMG $3301 \mathrm{~T}$ were also genotyped by PFGE, and fragment patterns were compared according to the criteria described by Tenover [14].

\section{MALDI-TOF MS and data analysis}

A single colony grown overnight on TSI (Triple Sugar Iron) agar was spotted in duplicate onto the MALDI target (MSP 96 target polished steel (MicroScoutTarget) plate; (Bruker Daltonik, Bremen, Germany) and air-dried at room temperature. After air-drying, each spot was overlaid with $1 \mu \mathrm{L}$ of HCCA (a-cyano-4-hydroxycinnamic acid) matrix solution saturated with organic solvent (50\% acetonitrile and $2.5 \%$ trifluoroacetic acid) and air-dried completely before MALDI-TOF MS. MALDITOF MS was carried out using a MALDI Microflex LT. Peptide mass fingerprint product ion spectra were acquired in linear positive mode at a laser frequency of $20 \mathrm{~Hz}$, within a mass range from 2000 to $20000 \mathrm{Da}$. For each spectrum, 240 laser shots were automatically acquired in 40 shot steps from different positions of the target spot (random walk movement) using AutoXecute acquisition control software (Flexcontrol 3.0; Bruker Daltonics, Bremen, Germany). The spectra were externally calibrated using the standard calibrant mixture (Escherichia coli extracts supplemented by proteins RNase A and myoglobin; Bruker Daltonics). To identify unknown bacteria, each peak list generated was matched directly against reference libraries (3502 species). Unknown spectra were compared with a library of reference spectra by means of a pattern-recognition algorithm making use of peak position, peak intensity distributions and peak frequencies. MALDI-TOF identifications were classified using modified versions of the score values proposed by the manufacturer: a score $\geq 2$ indicated species identification, a score in the range 1.7-1.99 indicated genus identification, and a score $<1.7$ denotes no identification. For the phylogenetic data analysis, a total of 16 spectra were automatically acquired with the AutoXecute acquisition control software for each strain (biological and technical replicates). MSP creation was carried out with the default setting of the Biotyper software (desired mass error for the MSP: 200; desired peak frequency minimum: 25\%; maximum desired peak number for the MSP: 70). Each Minimum spanning trees (MSP) was assigned to its specific node on the taxonomy tree. In order to visualize the relationship between the MSPs, dendrogram clustering was carried out using the standard settings of MALDI Biotyper software version 2.0 (distance measure: correlation; linkage: average). In addition, to evaluate the spectral variation within each strain, the composite correlation index (CCI) was computed by loading the raw data into the Biotyper software [15].

\section{Results}

\section{Phenotype analysis}

All isolated strains exhibited the same biochemical pattern (excellent identification: 99\%) and presented an overlapping antimicrobial susceptibility profile - they were all sensitive to gentamicin $(<1 \mu \mathrm{g} / \mathrm{ml})$, tobramycin $(<1 \mu \mathrm{g} / \mathrm{ml})$, amikacin $(16 \mu \mathrm{g} / \mathrm{ml})$, ciprofloxacin $(<0.25 \mu \mathrm{g} / \mathrm{ml})$, levofloxacin $(0.25 \mu \mathrm{g} / \mathrm{ml})$, imipenem $(2 \mu \mathrm{g} / \mathrm{ml})$, and sulfamethoxazole/ trimethoprim $(<20 \mu \mathrm{g} / \mathrm{ml})$, and resistant to ampicillin (>32 $\mu \mathrm{g} / \mathrm{ml})$, ampicillin/sulbactam $(>32 \mu \mathrm{g} / \mathrm{ml})$, cefazolin 
(>64 $\mu \mathrm{g} / \mathrm{ml})$, cefepime $(>64 \mu \mathrm{g} / \mathrm{ml})$, cefoxitine $(>64 \mu \mathrm{g} / \mathrm{ml})$, ceftazidime $(>64 \mu \mathrm{g} / \mathrm{ml})$, ceftriaxone $(>64 \mu \mathrm{g} / \mathrm{ml})$, piperacillin/tazobactam $(>128 \mu \mathrm{g} / \mathrm{ml})$ and nitrofurantoin $(256 \mu \mathrm{g} / \mathrm{ml})$. The negative Brucella agglutination sera test supported the biochemical identification.

\section{Rep-PCR-based DNA fingerprinting}

Genome fingerprinting was carried out using the DiversiLab System (bioMèrieux, France), which uses two interpretation criteria: Pearson correlation, based on the intensity of electrophoretic bands, and Kullbach correlation, focused on the

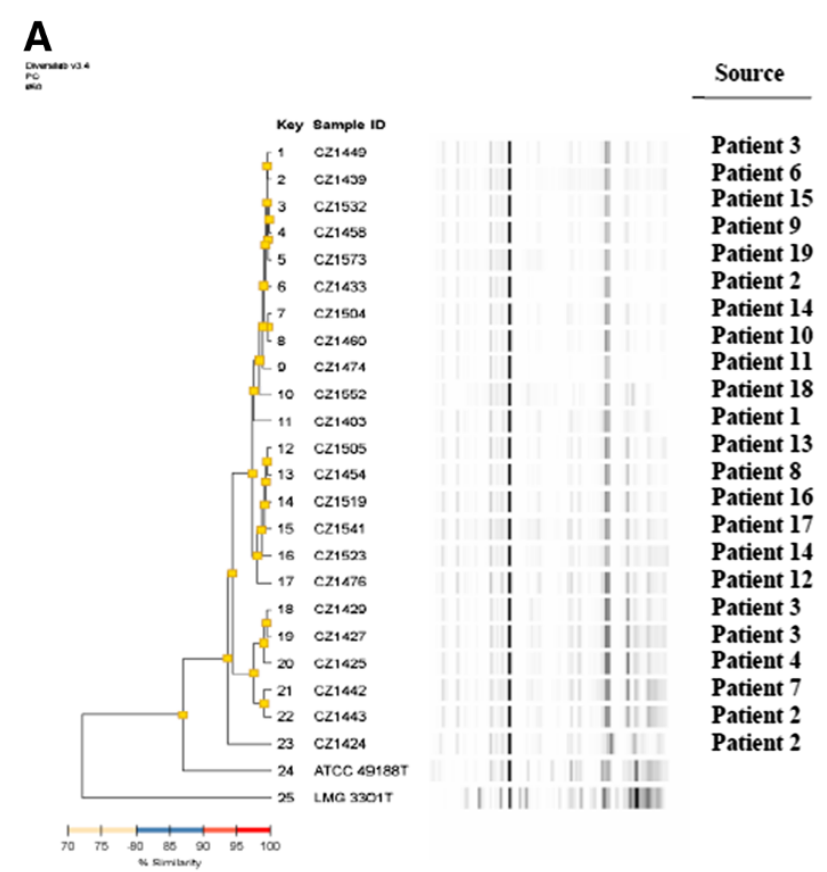

B

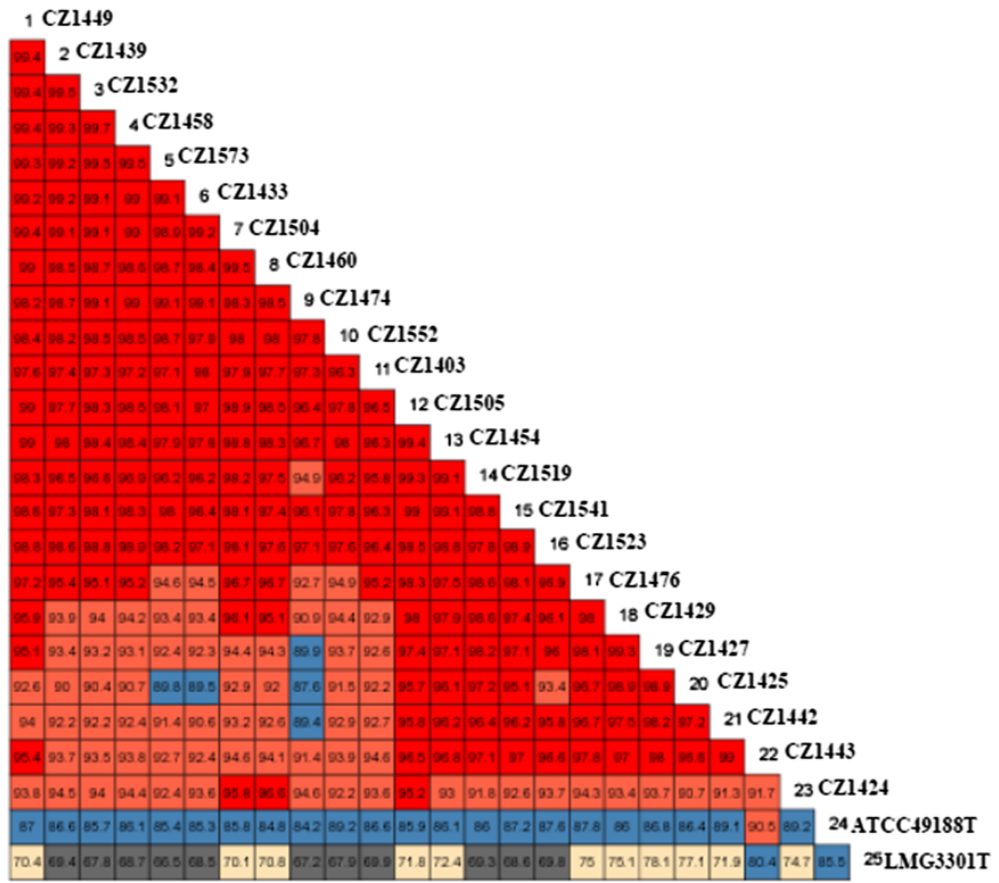

Figure 1 Dendrogram, virtual gel image (panel A) and similarity matrix (panel B) of 23 Ochrobactrum anthropi strains, 0 . anthropi ATCC 49188 T and $O$. intermedium LMG 3301 T, investigated by the DiversiLab System and further analyzed by Pearson correlation. (In Panel $\mathrm{B}$ the different colours and colour intensity refer to percentage of similarity). 
height and width of the peaks. Rep-PCR analysis identified four different patterns, as shown in the dendrogram in Figure 1 (panel A). Three rep-PCR patterns clustered isolates with $97 \%$ or more pattern similarity, and a further strain, CZ1424, showed a pattern of similarity of $<95 \%$. This strain showed a correlation index of $91.7 \%$ when compared with strain CZ1443, isolated from a different site in the same patient. Pearson correlation, associated to chronological evaluation of the clinical isolates, showed that strains found during the first timespan (from 26/04/2011 to 09/06/2011 as shown in Table 1) exhibited an overlap between 90 and 99\%, and were included in two different clusters (b and c). During the following timespan, up to the date of last bacterial isolation (24-08-2011), strain similarity was higher than 99\%; accordingly these bacteria were grouped in a single cluster (a). Unlike strains CZ1424 and CZ1443, bacterial strains isolated from the same patients from two different sites were similar or indistinguishable when their genome fingerprints were compared. In particular, CZ1427 and CZ1429 strains overlap by $99 \%$, CZ1429 and CZ1449 by $96 \%$ and CZ1427 and 1449 by $95.1 \%$. A similar behaviour was noted between strains CZ1504 and CZ1523 (98.1\% overlap) (Figure 1, panel B). In addition, as illustrated in Figure 1, panel B, all clinical strains investigated showed a pattern of similarity lower than $90.5 \%$ and $80.4 \%$ when compared to O. anthropi ATCC $49188 \mathrm{~T}$ and O. intermedium LMG $3301 \mathrm{~T}$ respectively. Kullback-Leibler analysis showed that the strains obtained later on in the outbreak, particularly 40 days after the first isolation, presented an inter-correlation greater than $92 \%$ (data not shown).

\section{PFGE data}

The 23 strains of $O$. anthropi were typed by digestion of the chromosomal DNA with SpeI endonuclease, and fragment separation was obtained by PFGE. Each pattern consisted of approximately 10-15 fragments, which were found to be identical to each other, except for strain $\mathrm{CZ}$
1552, whose 10-15-fragment pattern featured 6-7 fragment differences respect to the other pattern in the region between 145.5 and $485 \mathrm{Kbp}$. PFGE analysis thereby detected $22 / 23$ unique pulsotypes with a high degree of interrelatedness. O. anthropi ATCC $49188 \mathrm{~T}$ and O. intermedium LMG $3301 \mathrm{~T}$ appeared different from the 23 clinical isolates when compared according to Tenover's criteria (Figure 2).

\section{MALDI-TOF MS data}

A total of 46 spectra representing the 23 strains of $O$. anthropi were generated with the automated MALDITOF MS measurement. Protein mass patterns were detected in the mass range $2000-20,000 \mathrm{Da}$, were matched against Bruker Daltonics reference library, which included three $O$. anthropi ATCC strains, and resulted correctly identified at the species level (log score $\geq 2$ ). In order to create reliable MSPs for phylogenetic analysis, we measured a total of 368 spectra, 16 for each strain. Each mass spectrum dataset was compared with the others, yielding a matrix of cross-wise relatedness computed with the default setting provided by Biotyper 2.0 (CCI matrix). A CCI value approaching 1.0 showed confirmation of the set of spectra at a high level of significance, and is shown in Figure 3 by the brown squares at the diagonal intersection of the samples (maximum = self-to-self correlation). Inter-sample comparisons showed decreasing colour to yellow-blue, corresponding to decreasing degrees of correlation down to 0.02 , the lowest match. Composite correlation index analysis for the 23 Ochrobactrum anthropi strains showed similar inter-strain relatedness (Figure 3). Strains CZ1424 and CZ1443, as well as strains CZ1523 and CZ1504, isolated from the same patients but from two different sites, shared high degrees of similarity (over $80 \%$ and $85 \%$ respectively). Lower similarity, ranging from 60 to $80 \%$, was found among strains CZ1427, CZ1429 and CZ1449, also isolated from two different sites in the same patient. Strains CZ 1403, CZ1433 and CZ1442 showed the lowest degree of similarity with other strains (less than 20\%). At the other end of

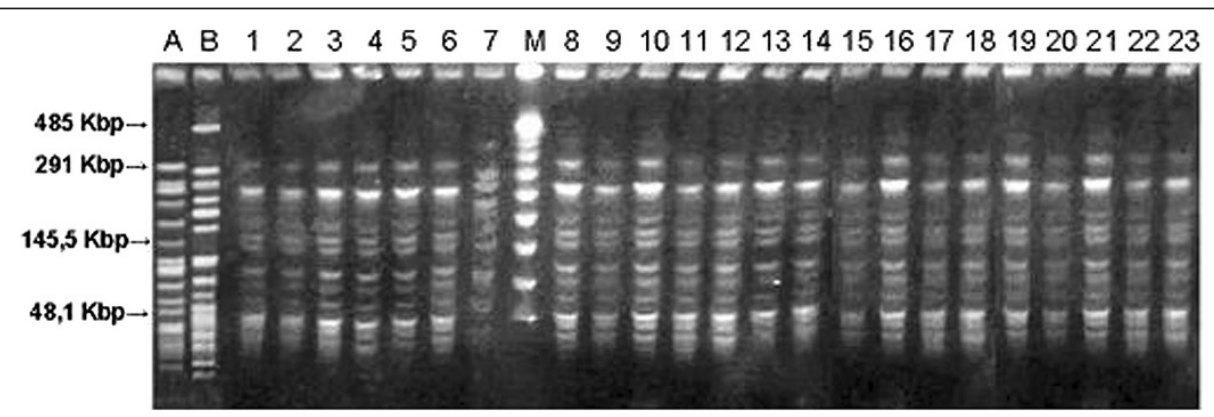

Figure 2 PFGE analysis of Ochrobactrum anthropi ATCC 49188 T (A), Ochrobactrum intermedium LMG 3301 T (B) and of 23 Ochrobactrum anthropi strains: (1)CZ1425,(2)CZ1424, (3)CZ1460, (4)CZ1442,(5)CZ1443,(6)CZ1449,(7)CZ1552,(M)molecular weight marker,(8)CZ1433,(9)CZ1504,(10)CZ1523,(11)CZ1427,(12)CZ1532(13)CZ1541,(14)CZ1439,(15)CZ1454,(16)CZ1519,(17)CZ1458,(18)CZ1476, (19)CZ1403, (20)CZ1573,(21)CZ1505,(22)CZ1474,(23)CZ1429. 


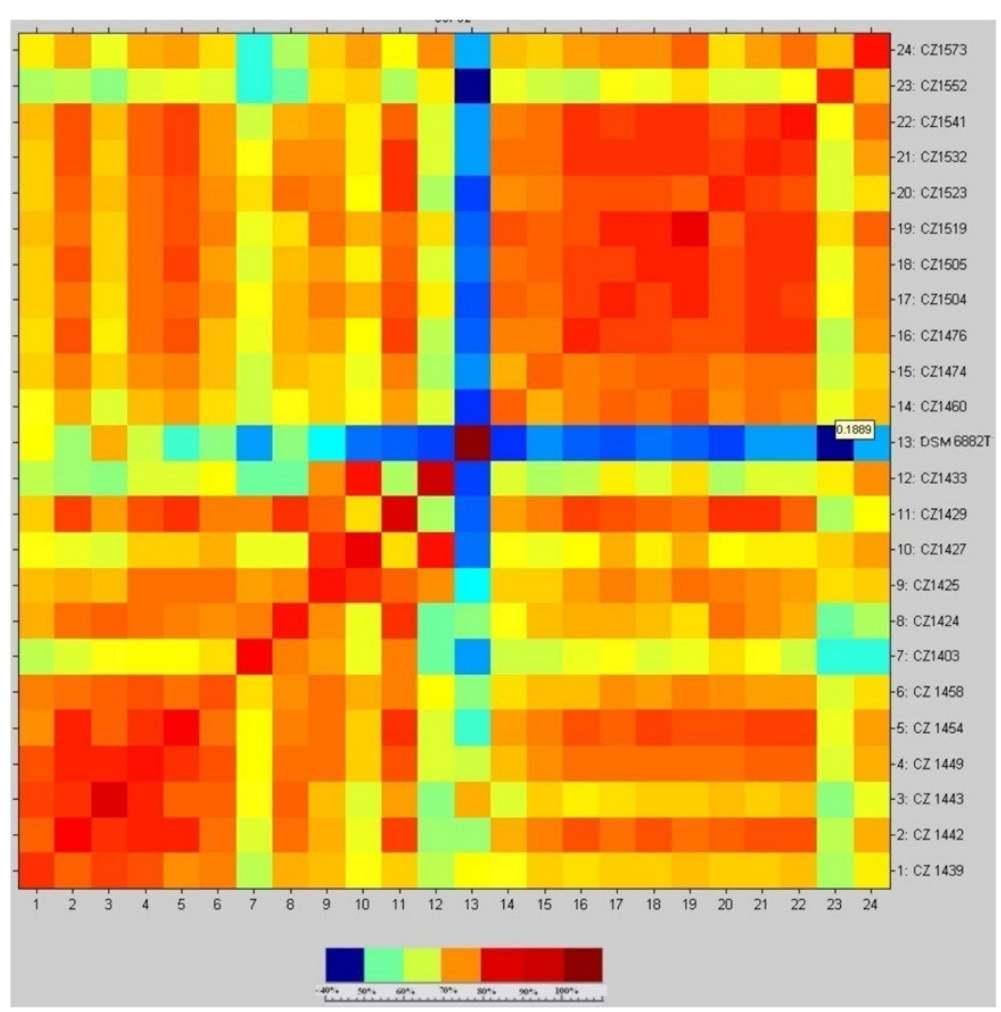

Figure 3 Composite correlation index $(\mathrm{CCl})$ matrix value for the strains of Ochrobactrum anthropi. Different colors indicate the correlation distance. CCI was calculated with MALDI Biotyper 2.0 software at the default settings: the lower boundary is 2000, the upper boundary is 20,000, the resolution of the mass range is four, and the number of intervals for $\mathrm{CCl}$ is four. A CCI value near 1.0 indicates relatedness between the spectral sets, and 0.02 indicates the lowest match.

the scale, two strain clusters (CZ1439, CZ1442, CZ1443, CZ1449, CZ1454, CZ1458 and CZ1460, CZ1474, CZ1476, CZ1504, CZ1505, CZ1519, CZ1523, CZ1532, CZ1541) shared a high degree of similarity (up to $95 \%$ ).

Based on the CCI data, a score-orientated MSP dendrogram was generated using the default setting of Biotyper
2.0, and included the 23 clinical strains and the 3 ATCC strains in the database (Figure 4). According to their mass signals and intensities, a hierarchic dendrogram clustered the 23 strains of $O$. anthropi in a single group, between 20 and 25 distance levels phylogenetically distinct from the ATCC isolates present in database. The dendrogram also

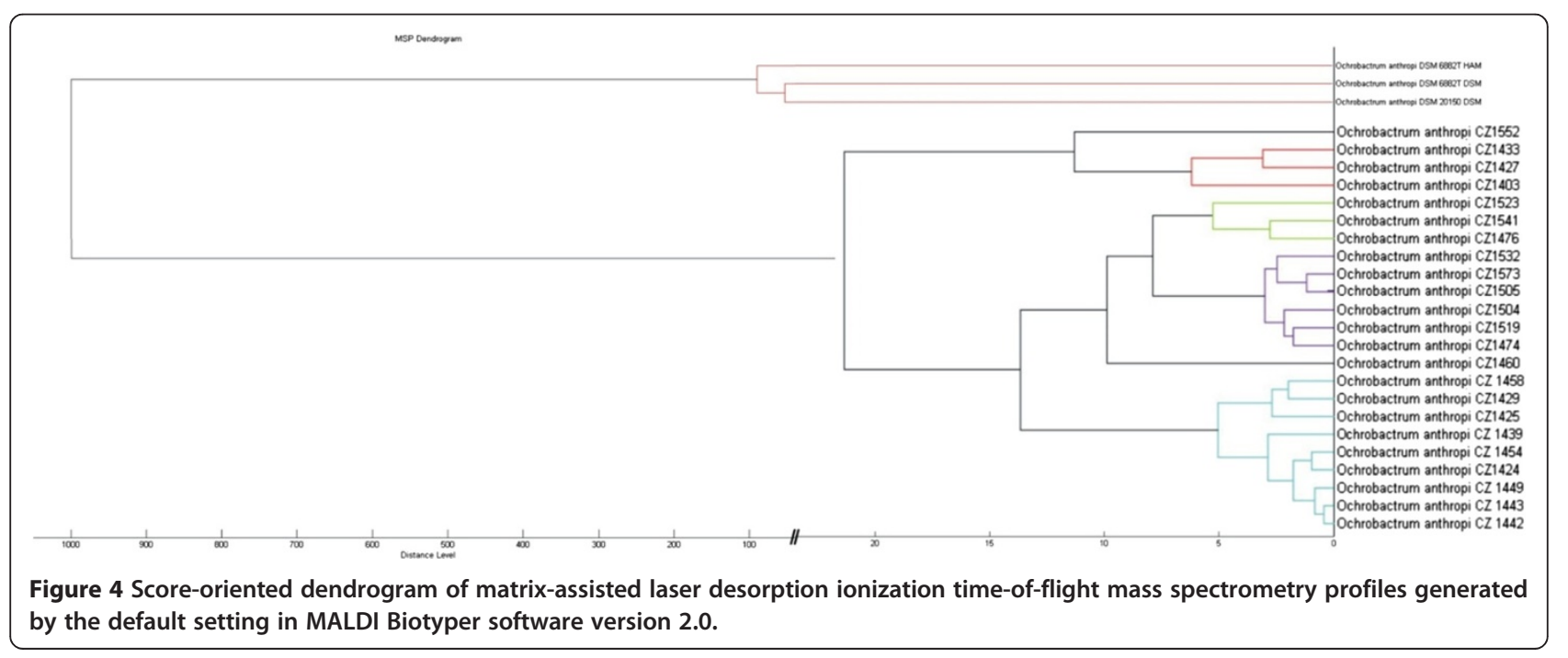


revealed the presence of four subgroups, each comprising the strains clustering at less than 5 distance levels, thereby indicating a high similarity between the isolates. Strains CZ1424 and CZ1443 were grouped in the same cluster with a distance level of up to 5, as were strains CZ1429 and CZ1449. Conversely, strains CZ1523 and CZ1504 were grouped in a different cluster, at a distance level greater than 10. Strain CZ1427, which showed a 60\% similarity with the other strains isolated from the same patient, was grouped at an inter-strain distance level of 10-15.

\section{Discussion}

$O$. anthropi is an adaptable bacterial species, whose individual strains can thrive in different environments. Indeed, after its molecular characterization [11] human-associated clonal complex data appear to indicate it possesses a specialized opportunistic behaviour [3]. It is frequently isolated from contaminated medical materials/devices and specimens obtained from immunocompromised patients [3], and after the first recognized case of human disease induced by this organism [16], O. anthropi infections causing primary or catheter-associated bacteraemia [1,17] have been increasingly reported [4]. With this in mind, when this infection did occur in our hospital, we set out to study the identification and typing of the $O$. anthropi strains through the genomic and proteomic correlation. To our knowledge, this represents the first study on strain typing of $O$. anthropi where the use of both rep-PCR and MALDI-TOF-MS-based fingerprinting were carried out. All patients developed infection during their stay in hospital, and in our Institution no cases of infection due to $O$. anthropi had been diagnosed before. Environmental and flushing solution cultures were negative for O. anthropi, therefore the source of the infection strains remained unclear. Fluoroquinolone monotherapy yielded good clinical response, however blood cultures from all patients became negative only after removal of CVC.

Our results indicate that all investigated strains were highly related and that they arose from a common ancestor, strongly providing evidence for a clonal origin of the infection. Interestingly, the strains detected early on during the outbreak showed a great variability in correlation range (90\%-99\%), while bacteria isolated later showed a correlation higher than $99 \%$. We can therefore speculate that $O$. anthropi is able to undergo rapid modifications, allowing bacteria to adapt to a human host. The proteomic profiles, which clustered the 23 strains in a single group, unrelated to the ATCC isolates present in the database (one of which comes from leech urine), further suggest a clonal origin of the infections. Moreover strains studied showed phylogenetically different patterns from ATCC reference strains used in all methods employed and different cluster patterns under rep-PCR fingerprinting and MALDI-TOF. A likely explanation for these differences could be that
rep-PCR analysis embraces the entire bacterial chromosome, whereas the main signals reported in MALDI-TOF MS are generated from ribosomal proteins alone $[18,13]$. Since we studied a small number of strains, we can't draw firm conclusions about the correlation between automated rep-PCR and MALDI-TOF for molecular typing of Ochrobactrum anthropi. However, both methods have demonstrated a similar sensitivity in discriminating the variability among the strains studied. Although strict comparison between PFGE and MALDI-TOF was problematic, due to the different methods involved (i.e., protein profiling for MALDI-TOF dendrogram and genetic profiling for PFGE), the tests showed a similar separation between the CZ1552 strain and the other strains. Although the results obtained by the two techniques were similar, on the whole, MALDI-TOF results were obtained much more rapidly, within a few minutes. MALDI-TOF is not only much easier and less-time consuming than PFGE, it also requires a limited amount of bacterial colonies and allows comparison at all times with the universal database.

Semi-automated rep-PCR appeared to be more discriminative than PFGE in typing the $23 \mathrm{O}$. anthropi strains isolated during this hospital outbreak. Both repPCR and MALDI-TOF MS yielded four clusters and a common ancestor, while PFGE showed the same PFGE profile in 22 isolates. In PFGE, strain CZ1552 was the odd one out, whereas rep-PCR identified strain CZ1424 as being different. These strains were found to be genetically unrelated to each other.

The marker used for the rep-PCR analysis (the region between the noncoding repetitive sequences in bacterial genomes) is less genetically stable than the one used for PFGE (the target sequence of the SpeI restriction enzyme). Hence, the variability shown by rep-PCR is likely to represent changes in the same clone that could not be detected by PFGE [19]. Rep-PCR analysis is a technique aimed at defining clonal relationships, and its ease of use and faster turnaround time as compared to PFGE makes it a rapid method of screening outbreaks of O. anthropi and therefore allows timely implementation of control measures.

\section{Conclusions}

In conclusion, rep-PCR and MALDI-TOF MS appear to be extremely useful for evaluation of clonal relationships between isolates. The different marker (genomic vs. proteomic) evaluated, as well as the completely different techniques used increase the reliability with which isolate similarity or diversity may be assessed during a hospital outbreak. In addition, we believe that advances in the molecular typing of Ochrobactrum anthropi would facilitate the study on the epidemiology, prevention and control of the infections caused by this pathogen. 


\section{Abbreviations}

O. anthropi: Ochrobactrum anthropi; rep-PCR: Repetitive extragenic palindromic-polymerase chain reaction; MALDI-TOF: Matrix-assisted laser desorption/ionization-time-of-flight; PFGE: Pulsed-field gel electrophoresis; UPGMA: Unweighted pair group method with arithmetic mean; CVC: Central venous catheter; $O$. intermedium: Ochrobactrum intermedium; TSI: Triple Sugar Iron; HCCA: a-cyano-4-hydroxycinnamic acid; MSP: Minimum spanning trees; CCl: Composite correlation index.

\section{Competing interests}

The study was supported by Dept of Health Sciences, "Magna Graecia" University of Catanzaro. None of the authors has a financial relationship with other people or organizations that could inappropriately influence its findings.

\section{Authors' contributions}

AQ participated in the design of the study, drafted the manuscript and carried out automated repetitive extragenic palindromic-polymerase chain reaction, GP carried out MALDI-TOF MS and PFGE analysis and contributed in the draft of the manuscript, ' LR carried out automated repetitive extragenic palindromic-polymerase chain reaction, RP and NM carried out bacteriological cultures and identification of microorganisms, MRC participated and coordinated study on proteomic analysis, GM participated in the design and contributed in the draft and editing of the manuscript, MCL participated in the design and coordination of the study and contributed in the draft and editing of the manuscript, AF conceived the study and participated in its design and coordination. All authors read and approved the final manuscript.

\section{Author details}

IInstitute of Microbiology, Department of Health Sciences, "Magna Graecia" University, Viale Europa, Catanzaro, Italy. ${ }^{2}$ Department of Molecular and Cellular Biology and Pathology L. Califano, Medicine School, University of Naples Federico II, Naples, Italy.

Received: 5 September 2013 Accepted: 18 March 2014

Published: 22 March 2014

\section{References}

1. Soloaga R, Carrion N, Pidone J, Guelfand L, Margari A, Altieri R: Catheterassociated bacteremia caused by Ochrobactrum anthropi. Medicina (B Aires) 2009, 69(suppl.6):655-657.

2. Nadjar D, Labia R, Cerceau C, Bizet C, Philippon A, Arlet G: Molecular characterization of chromosomal class $\mathrm{C}$ beta-lactamase and its regulatory gene in Ochrobactrum anthropi. Antimicrob Agents Chemother 2001, 45(suppl.8):2324-2330.

3. Romano S, Aujoulat F, Jumas-Bilak E, Masnou A, Jeannot JL, Falsen E, Marchandin $\mathrm{H}$, Teyssier $\mathrm{C}$ : Multilocus sequence typing supports the hypothesis that Ochrobactrum anthropi displays a human-associated subpopulation. BMC Microbio/ 2009, 9:267. doi:10.1186/1471-2180-9-267.

4. Daxboeck F, Zitta S, Assadian O, Krause R, Wenisch C, Kovarik J: Ochrobactrum anthropi bloodstream infection complicating hemodialysis. Am J Kidney Dis 2002, 40(suppl. 4):E17.

5. Shrishrimal K: Recurrent: Ochrobactrum anthropi and Shewanella putrefaciens bloodstream infection complicating hemodialysis. Hemodial Int 2011. doi:10.1111/j.1542-4758.2011.00586.x.

6. Wi YM, Peck KR: Biliary sepsis caused by Ochrobactrum anthropi. Jpn J Infect Dis 2010, 63(suppl.6):444-446.

7. Song S, Ahn JK, Lee GH, Park YG: An epidemic of chronic pseudophakic endophthalmitis due to Ochrobactrum anthropi: clinical findings and managements of nine consecutive cases. Ocul Immunol Inflamm 2007, 15(suppl.6):429-434.

8. van Dijck P, Delmée M, Ezzedine H, Deplano A, Struelens MJ: Evaluation of pulsed-field gel electrophoresis and rep-PCR for the epidemiological analysis of Ochrobactrum anthropi strains. Eur J Clin Microbiol Infect Dis 1995, 14(suppl.12):1099-1102.

9. Naik C, Kulkarni H, Darabi A, Bhanot N: Ochrobactrum anthropi: a rare cause of pneumonia. J Infect Chemother 2013, 19(1):162-5.

10. Lebuhn M, Achouak W, Schloter M, Berge O, Meier H, Barakat M, Hartmann A, Heulin T: Taxonomic characterization of Ochrobactrum sp. Isolates from soi samples and wheat roots, and description of Ochrobactrum tritici sp. nov. and Ochrobactrum grignonense sp. nov. Int J Syst Evol Microbiol 2000, 50:2207-2223.

11. Bathe S, Achouak W, Hartmann A, Heulin T, Schloter M, Lebuhn M: Genetic and phenotypic microdiversity of Ochrobactrum spp. FEMS Microbiol Ecol 2006, 56(suppl.2):272-280.

12. Bizzini A, Jaton K, Romo D, Bille J, Prod'hom G, Greub G: MALDI-TOF Mass Spectrometry as an alternative to $16 \mathrm{~S}$ rDna sequencing for identification of difficult to identify bacterial strains. J Clin Microbio/ 2010. doi:10.1128/ JCM.01463-10.

13. Treviño M, Navarro D, Barbeito G, García-Riestra C, Crespo C, Regueiro BJ: Molecular and epidemiological analysis of nosocomial carbapenem-resistant Klebsiella spp. using repetitive extragenic palindromic-polymerase chain reaction and matrix-assisted laser desorption/ionization-time of flight. Microb Drug Resist 2011, 17(3):433-442. doi:10.1089/mdr.2010.0182.

14. Tenover FC, Arbeit RD, Goering RV, Mickelsen PA, Murray BE, Persing DH, Swaminathan B: Interpreting chromosomal DNA restriction patterns produced by pulsed-field gel electrophoresis: criteria for bacterial strain typing. J Clin Microbiol 1995, 33:2233-2239.

15. Pulcrano G, Roscetto E, lula VD, Panellis D, Rossano F, Catania MR: MALDITOF mass spectrometry and microsatellite markers to evaluate Candida parapsilosis transmission in neonatal intensive care units. Eur J Clin Microbiol Infect Dis 2012, 31:2919-2928.

16. Appelbaum PC, Campbell DB: Pancreatic abscess associated with Achromobacter group Vd biovar 1. J Clin Microbiol 1980, 12:282-283.

17. Cieslak TJ, Robb ML, Drabick CJ, Fischer GW: Catheter-associated sepsis caused by Ochrobactrum anthropi: report of a case and review of related nonfermentative bacteria. Clin Infect Dis 1992, 14(suppl.4):902-907.

18. Treviño M, Navarro D, Barbeito G, Areses P, García-Riestra C, Regueiro BJ: Plasmid-mediated AMPc producing Proteus mirabilis in the Health Care Area of Santiago de Compostela: molecular and epidemiological analysis by rep-PCR and MALDI-TOF. Rev Esp Quimioter 2012, 25(2):122-8.

19. Ligozzi M, Fontana R, Aldegheri M, Scalet G, Lo Cascio G: Comparative evaluation of an automated repetitive-sequence-based PCR instrument versus pulsed-field gel electrophoresis in the setting of a Serratia marcescens nosocomial infection outbreak. J Clin Microbiol 2010, 48(5):1690-5.

doi:10.1186/1471-2180-14-74

Cite this article as: Quirino et al:: Typing of Ochrobactrum anthropi clinical isolates using automated repetitive extragenic palindromicpolymerase chain reaction DNA fingerprinting and matrix-assisted laser desorption/ionization-time-of-flight mass spectrometry. BMC Microbiology 2014 14:74.

\section{Submit your next manuscript to BioMed Central and take full advantage of:}

- Convenient online submission

- Thorough peer review

- No space constraints or color figure charges

- Immediate publication on acceptance

- Inclusion in PubMed, CAS, Scopus and Google Scholar

- Research which is freely available for redistribution 